\title{
Erratum: Spin-orbit interaction driven collective electron-hole excitations in a noncentrosymmetric nodal loop Weyl semimetal [Phys. Rev. B 92, 115149 (2015)]
}

Kyo-Hoon Ahn, Kwan-Woo Lee, and Warren E. Pickett

(Received 22 September 2016; published 30 September 2016)

DOI: 10.1103/PhysRevB.94.119902

The scale of the loss function in Fig. 6 of our paper (Fig. 1 here) should be multiplied by a factor of 2. This issue arises when calculating the dielectric function with the WIEN2K code. For a noncentrosymmetric crystal structure when spin-orbit coupling is included, it is necessary to take into account a factor of 2 not provided by the code. Only the magnitude is affected, while the energy dependence is not, thus our conclusions are unchanged.

We thank N. Armstrong for providing his unpublished experimental results and useful communications.

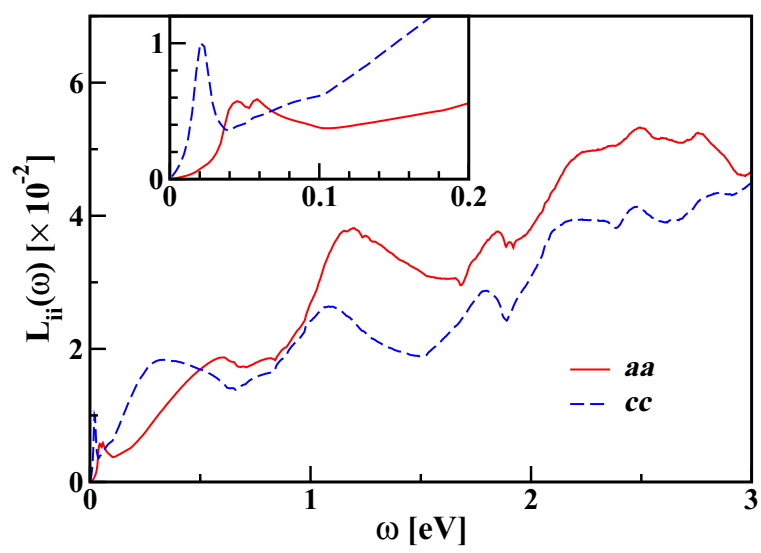

FIG. 1. The energy-loss functions are multiplied by a factor of 2 . 